\title{
Stark broadening of Cd I spectral lines ${ }^{\star}$
}

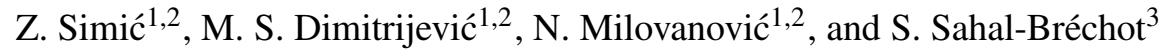 \\ 1 Astronomical Observatory, Volgina 7, 11160 Belgrade, Serbia and Montenegro \\ e-mail: [zsimic;mdimitrijevic]@aob.bg.ac.yu \\ 2 Institute Isaac Newton of Chile Yugoslavia Branch, and Universidad Diego Portales, Chile \\ 3 Observatoire de Paris-Meudon, 92195 Meudon, France
}

Received 14 January 2005 / Accepted 7 March 2005

\begin{abstract}
Stark broadening parameters, widths, and shifts for $33 \mathrm{Cd}$ I singlets and 37 triplets were calculated using the semiclassical perturbation method. The results were compared with available experimental and theoretical data. Also, regularity in the spectral series $5 \mathrm{~s}^{2}{ }^{1} \mathrm{~S}-\mathrm{np}{ }^{1} \mathrm{P}^{\circ}$ was investigated. The influence of Stark broadening was analyzed in A-type stellar atmospheres.
\end{abstract}

Key words. line: profiles - atomic data - atomic processes - lines formation - stars: atmospheres

\section{Introduction}

Cadmium lines are interesting due to their presence in stellar atmospheres. They have been identified in A-type star spectra, as e.g. 68 Tauri (Adelman 1994a,b), $\chi$ Lupi (Leckrone et al. 1999), V816 Centauri (Cowley et al. 2000). As an example Adelman has shown for $68 \mathrm{Tau}$ that the $[\mathrm{Cd} / \mathrm{H}]$ value $(-6.57 \pm 0.15)$ is very large in comparison to the one for the Sun $(-10.14)$. It is worth noting that for the atmosphere modelisation of this star with $T_{\text {eff }}=9025 \mathrm{~K}$ and $\log g=3.95$ (Adelman 1994a,b), Stark broadening data for C II, Mg II, Si II, and Ca II lines (Sahal-Bréchot 1969a,b; Chapelle \& Sahal-Bréchot 1970; Lanz et al. 1988) have been used, which shows the usefulness of such data for A-type star spectra analysis.

The particularity of neutral cadmium is that the line $6438.4696 \AA$, $5 \mathrm{p}{ }^{1} \mathrm{P}_{1}^{\circ}-5 \mathrm{~d}^{1} \mathrm{D}_{2}$ is the fundamental wavelength on which other standards are based. Moreover, neutral cadmium is important in analytical spectrochemistry because of its significance in toxicological and environmental studies.

The first experimental investigations of the influence of the Stark broadening mechanism on cadmium lines were performed by Nagibina (1958) and by Gorodnichyute \& Gorodnichyus (1961). Here, the experimental results obtained by Kusch \& Oberschelp (1967) are of interest. Also, the first theoretical determination was by Grechikhin (1969), and in our case we will use the results obtained by Dimitrijević \& Konjević (1983) for comparison.

Stark broadening of cadmium lines is also useful when considering of regularities and systematic trends, and the

* Tables 1 and 2 are only available in electronic form at the CDS via anonymous ftp to cdsarc.u-strasbg.fr (130.79.128.5) or via http://cdsweb.u-strasbg.fr/cgi-bin/qcat?J/A+A/441/391 corresponding results may be of interest in astrophysics for interpolation of new data and critical evaluation of existing ones. We note here that the first investigation of regularity in spectral series was that of Wiese \& Konjević (1982).

Here, we use the semiclassical perturbation approach (Sahal-Bréchot 1969a,b) to calculate the Stark broadening parameters of $33 \mathrm{Cd}$ I singlets and 37 triplets as a function of electron density for temperatures between $2500 \mathrm{~K}$ and $50000 \mathrm{~K}$, which are particularly interesting for stellar investigation. The results obtained are then used for analysing of the influence of Stark broadening in A-type stellar atmospheres.

\section{Results and discussion}

For neutral cadmium lines Stark broadening parameters (the full line width at half maximum $-W$ and the line shift $-d$ ) were calculated by using the semiclassical perturbation formalism (Sahal-Bréchot 1969a,b). This formalism, as well as the corresponding computer code, has been updated and optimized several times (Sahal-Bréchot 1974, 1991; Fleurier et al. 1977; Dimitrijević \& Sahal-Bréchot 1984; Dimitrijević et al. 1991; Dimitrijević \& Sahal-Bréchot 1996). A brief review of the calculation procedure, with discussion of updatings and validity criteria is given by Dimitrijević (1996). The atomic energy levels needed for the calculations were taken from Moore (1971). The oscillator strengths were calculated within the Coulomb approximation (Bates \& Damgaard 1949; and the tables of Oertel \& Shomo 1968). For higher levels, the method of van Regemorter et al. (1979) was used.

In Tables 1 and 2 (available only in the electronic form at the CDS), electron-, proton-, and He II-impact broadening parameters for $33 \mathrm{CdI}$ singlets (Table 1) and $37 \mathrm{Cd}$ I triplets (Table 2) for perturber densities from $10^{13} \mathrm{~cm}^{-3}$ up to 
Table 3. Experimental Stark widths $-W_{m}$, our theoretical results $W_{\mathrm{th}}$, theoretical results within GBKO approach $-W_{\mathrm{th}^{\prime}}$.

\begin{tabular}{ccccc}
\hline \hline TRANSITION & $\lambda(\AA)$ & $W_{m}(\AA)$ & $W_{m} / W_{\text {th }}$ & $W_{m} / W_{\mathrm{th}^{\prime}}$ \\
\hline CdI & 5085.8 & 3.67 & 6.41 & 6.34 \\
& 4799.9 & 3.84 & 7.53 & 6.63 \\
$5 \mathrm{p}^{3} \mathrm{P}^{\circ}-6 \mathrm{~s}^{3} \mathrm{~S}^{\circ}$ & 4678.2 & 1.74 & 3.59 & 3.00 \\
\hline
\end{tabular}

$10^{19} \mathrm{~cm}^{-3}$ along with temperatures from 2500 to $50000 \mathrm{~K}$, are shown. Parameter C (Dimitrijević \& Sahal-Bréchot 1984) is also given as an estimate for the maximum perturber density for which the line may be treated as isolated, obtained when $\mathrm{C}$ is divided by the corresponding full width at half maximum. For perturber densities lower than the lowest tabulated value, the behavior of the corresponding Stark broadening parameters is linear. The validity of the impact approximation was estimated for data shown in Tables 1 and 2, by checking if the collision volume $(V)$ multiplied by the perturber density $(N)$ is much less than one (Sahal-Bréchot 1969a,b), and in all cases consider the product $N V$ is less than 0.1 . When the impact approximation is not valid, the ion broadening contribution may be estimated by using the quasistatic approach (Sahal-Bréchot 1991; or Griem 1974). In the region between, where neither of these two approximations is valid, a unified type theory should be used. For example in Barnard et al. (1974), a simple analytical formula for such a case is given. The accuracy of the results obtained decreases when broadening by ion interactions increases.

We compare our results (see Table 3 ) for Cd I $5 \mathrm{p}^{3} \mathrm{P}^{\circ}-6 \mathrm{~s}^{3} \mathrm{~S}^{\circ}$ multiplet with existing experimental data (Kusch \& Oberschelp 1967). In this experiment Stark widths were determined by using spark discharge tube with $\mathrm{Cd}\left(\mathrm{CH}_{3}\right)_{2}$ and $\mathrm{Cd}\left(\mathrm{C}_{2} \mathrm{H}_{5}\right)_{2}$ for perturber density normalized at value of $10^{17} \mathrm{~cm}^{-3}$ and for temperature of $11100 \mathrm{~K}$. Also, for the same multiplet there are theoretical results obtained within GBKO approach (Griem et al. 1962) by Dimitrijević \& Konjević (1983). Both theoretical results disagree with experimental ones. Konjević et al. (1984a) indicate selfabsorption as a possible reason for this.

In cases where there are no reliable data for Stark broadening, investigation of regularities and systematic trends can quickly estimate missing values, especially if it is not necessary to have high accuracy for each particular line and good accuracy for a large number of lines is sufficient (see e.g. Purić et al. 1980, 1991; Dimitrijević 1982; Wiese \& Konjević 1982, 1992; Tankosić et al. 2001).

The regular behavior of spectral line Stark widths in spectral series has been shown first by Wiese \& Konjević (1982). This was also investigated by Dimitrijević \& Sahal-Bréchot (1984, 1996), Tankosić et al. (2001), and Dimitrijević (2003, 2004). One of the goals is to investigate regularity within a spectral series of $\mathrm{Cd}$ I and then obtain an analytical expression that can be useful for estimating new data.

Sarandev et al. (2000) developed an approximate method for estimation of Stark spectral line widths and applied to the determination of spectral line widths for several heavy ions such as $\mathrm{Cd}$ I, Ge I, $\mathrm{Hg}$ I, Pb I, Rb I, SnI, Zn I. Estimates were done for $T=20000 \mathrm{~K}$ and $N_{\mathrm{e}}=10^{17} \mathrm{~cm}^{-3}$. For $5 \mathrm{p}{ }^{3} \mathrm{P}^{\circ}-$ $6 \mathrm{~s}{ }^{3} \mathrm{~S}$ transition of $\mathrm{Cd} \mathrm{I}$, they obtained full line width at half

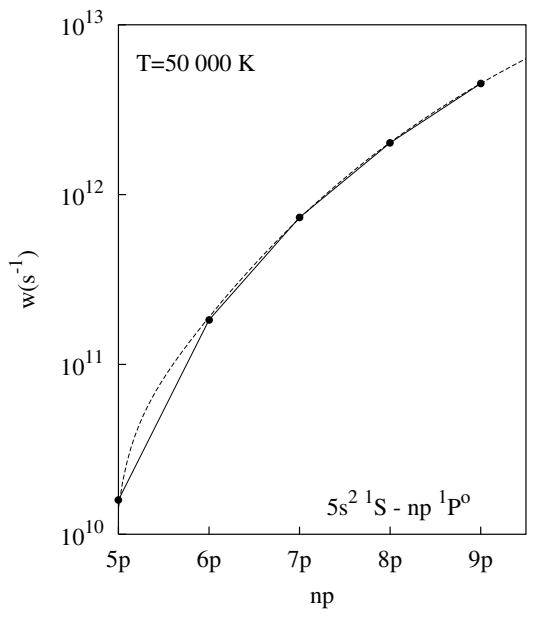

Fig. 1. Electron-impact widths for Cd I $5 s^{2}{ }^{1} \mathrm{~S}-n \mathrm{p}{ }^{1} \mathrm{P}^{\circ}$ spectral series in angular frequency units as a function of main quantum number $n$ for the upper atomic energy level.

maximum $W=0.58 \AA$, which is very close to the semiclassical result of Dimitrijević \& Konjević (1983), $W=0.62 \AA$, as well as to our result $W=0.47 \AA$.

In Fig. 1 electron-impact full half widths $W$ in angular frequency units, for $\mathrm{CdI} 5 \mathrm{~s}^{2}{ }^{1} \mathrm{~S}-n \mathrm{p}{ }^{1} \mathrm{P}^{\circ}$ lines are shown as a function of quantum number $n$, for $T=50000 \mathrm{~K}$ at $N_{\mathrm{e}}=$ $10^{16} \mathrm{~cm}^{-3}$. We see a gradual changing in Stark widths within the $5 \mathrm{~s}^{2}{ }^{1} \mathrm{~S}-n \mathrm{p}{ }^{1} \mathrm{P}^{\circ}$ spectral series. Such regular behavior of Stark widths is the consequence of the gradual change in the energy separations between the initial (upper) level and the principal perturbing levels. This function $W(n)$ has been interpolated using the third power polynominal

$W(n)=a n^{3}+b n^{2}+c n+d$.

Here $W$ is a full width at half maximum $(F W H M)$ expressed in $\mathrm{rad} / \mathrm{s}$ per electron, and constants are $a=6.83417 \times 10^{10}$, $b=-1.05083 \times 10^{12}, c=5.15558 \times 10^{12}$, and $d=-9.83561 \times$ $10^{12}$. The polynomial function is represented by a dotted line in Fig. 1. Using the previous expression, it is estimated that the Stark width for the Cd I $5 \mathrm{~s}^{2}{ }^{1} \mathrm{~S}-10 \mathrm{p}{ }^{1} \mathrm{P}^{\circ}$ spectral line for which there is no enough atomic data is $W=8.564 \times 10^{12} \mathrm{~s}^{-1}$ i.e. $W=0.911 \AA$.

One of our aims here is to use the results to investigate the influence of Stark broadening within a spectral series in A-type stellar atmospheres. Consequently, Stark widths within the $5 \mathrm{~s}^{2}{ }^{1} \mathrm{~S}-\mathrm{np}{ }^{1} \mathrm{P}^{\circ}$ spectral series were compared in Fig. 2 with Doppler widths for a model $\left(T_{\text {eff }}=10000 \mathrm{~K}, \log g=4\right)$ of an A-type stellar atmosphere (Kurucz 1979), close to the conditions for 68 Tauri $\left(T_{\text {eff }}=9025 \mathrm{~K}, \log g=3.95\right)$ where Stark broadening is of interest for the atmosphere modelisation (Adelman 1994a,b). We note also that one of the lines (2288.7 $\AA$ ) within the first member of this series, the multiplet $5 \mathrm{~s}^{2}{ }^{1} \mathrm{~S}-5 \mathrm{p}{ }^{1} \mathrm{P}^{\circ}$, has an intensity of 1500 according to the NIST Atomic Spectra Database. Our results are presented as a function of Rosseland optical depth $-\log \tau$. With an increasing the principal quantum number the importance of Stark broadening in comparison to the Doppler width increases as well. For lines 


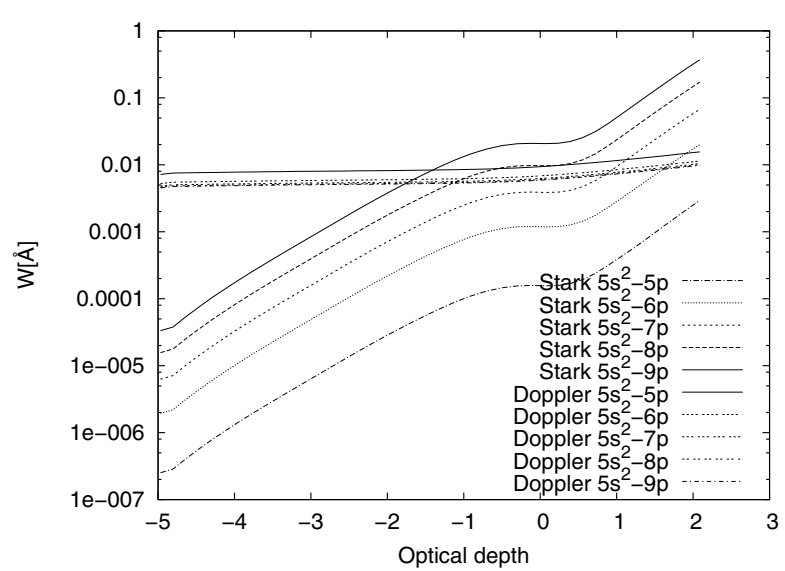

Fig. 2. Thermal Doppler and Stark widths for CdI singlet spectral lines: $5 \mathrm{~s}^{2}{ }^{1} \mathrm{~S}-5 \mathrm{p}{ }^{1} \mathrm{P}^{\circ}(2288.7 \AA), 5 \mathrm{~s}^{2}{ }^{1} \mathrm{~S}-6 \mathrm{p}{ }^{1} \mathrm{P}^{\circ}(1669.3 \AA)$, $5 \mathrm{~s}^{2}{ }^{1} \mathrm{~S}-7 \mathrm{p}{ }^{1} \mathrm{P}^{\circ}(1526.9 \AA), 5 \mathrm{~s}^{2}{ }^{1} \mathrm{~S}-8 \mathrm{p}{ }^{1} \mathrm{P}^{\circ}(1469.4 \AA), 5 \mathrm{~s}^{2}{ }^{1} \mathrm{~S}-9 \mathrm{p}{ }^{1} \mathrm{P}^{\circ}$ (1440.2 $\AA$ ) as a function of optical depth.

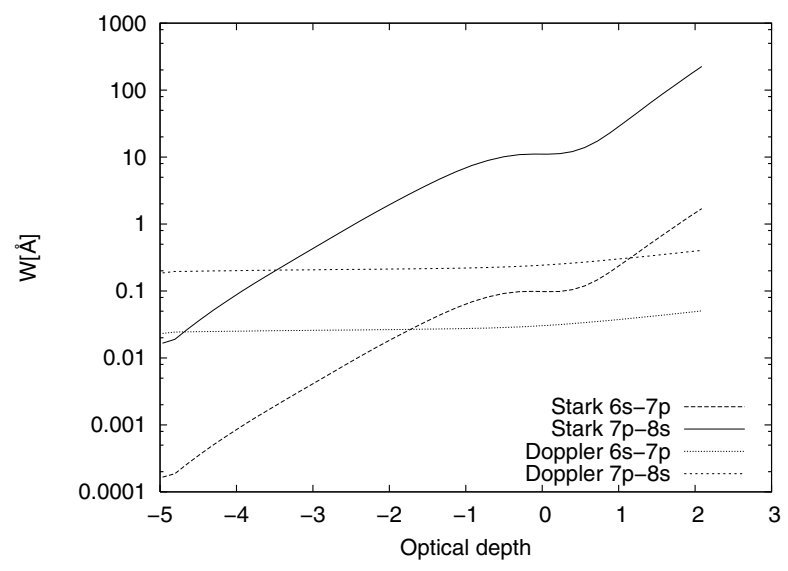

Fig. 3. Thermal Doppler and Stark widths for Cd I triplet spectral line: $6 \mathrm{~s}^{3} \mathrm{~S}^{\circ}-7 \mathrm{p}^{3} \mathrm{P}^{\circ}(7400.9 \AA)$ and $7 \mathrm{p}^{3} \mathrm{P}^{\circ}-8 \mathrm{~s}^{3} \mathrm{~S}^{\circ}(59346.5 \AA)$ as a function of optical depth.

with higher initial quantum number, Stark broadening is more than one magnitude larger than the Doppler mechanism.

This model for the stellar atmosphere has been used for two other spectral lines, the first $(7400.9 \AA)$ in the optical range and the second (59346.5 $\AA$ ) in the IC range (see Fig. 3 ). It is interesting to see in Fig. 3, that the Stark broadening mechanism is absolutely dominant in comparison with the thermal Doppler mechanism throughout the considered stellar atmosphere layers $(\log \tau>-3.5)$ for the $59346.5 \AA$ line. This is the consequence of the fact that infrared spectral lines originate from transitions with longer wavelengths than the lines in UV and optical ranges, and the corresponding Stark broadening, proportional to the square of the wavelength, is larger in comparison to the Doppler one, which is proportional to $\lambda$.

For the atmosphere model considering, the Stark broadening effect should be taken into account in abundance determinations and other investigations of stellar plasmas.

Acknowledgements. This work is part of the project GA 1195 "Influence of collisional processes on astrophysical plasma lineshapes", supported by the Ministry of Science and Environment
Protection of Serbia. The research was also supported by the Fonds zur förderung der wissenschaftlichen Forschung (Project S7303-AST).

\section{References}

Adelman, S. J. 1994a, MNRAS, 266, 97

Adelman, S. J. 1994b, MNRAS, 271, 355

Barnard, A. J., Cooper, J., \& Smith, E.W. 1974, J. Quant. Spectrosc. Radiative Transfer, 14, 1025

Bates, D. R., \& Damgaard, A. 1949, Phil. Trans. Roy. Soc. London, Ser. A, 242, 101

Cowley, C. R., Ryabchikova, T., Kupka, F., et al. 2000, MNRAS, 317 , 299

Chapelle, J., \& Sahal-Bréchot, S. 1970, A\&A, 6, 415

Dimitrijević, M. S. 1982, A\&A, 112, 251

Dimitrijević, M. S. 1996, Zh. Prikl. Spektrosk., 63, 810

Dimitrijević, M. S., \& Konjević, N. 1983, J. Quant. Spectrosc. Radiat. Transfer, 30, 45

Dimitrijević, M. S., \& Sahal-Bréchot, S. 1984, J. Quant. Spectrosc. Radiat. Transfer, 31, 301

Dimitrijević, M. S., \& Sahal-Bréchot, S. 1996, Phys. Scr., 54, 50

Dimitrijević, M. S., Sahal-Bréchot, S., \& Bommier, V. 1991, A\&AS, 89,581

Dimitrijević, M. S., Dačić, M., Cvetković, Z., \& Sahal-Bréchot, S. 2003, A\&A, 400, 791

Dimitrijević, M. S., Dačić, M., Cvetković, Z., \& Simić, Z. 2004, A\&A, 425,1147

Fleurier, C., Sahal-Bréchot, S., \& Chapelle, J. 1977, J. Quant. Spectrosc. Radiative Transfer, 17, 595

Gorodnichyute, M. G., \& Gorodnichyus, G. A. 1961, Litov. Fiz. Sb., 1,163

Grechikhin, L. I. 1969, J. Appl. Spectrosc., 11, 870

Griem, H. R. 1974, Spectral Line Broadening by Plasmas (New York: Academic Press)

Griem, H. R., Baranger, M., Kolb, A. C., \& Oertel, G. K. 1962, Phys. Rev., 125, 177

Konjević, N., Dimitrijević, M. S., \& Wiese, W. L. 1984a, J. Phys. Chem. Ref. Data, 13, 619

Kurucz, R. L. 1979 ApJS, 40, 1

Kusch, H. J., \& Oberschelp, E. 1967, Z. Astrophys., 67, 77

Lanz, T., Dimitrijević, M. S., \& Artru, M.-C. 1988, A\&A, 192, 249

Leckrone, D. S., Proffitt, C. R., Wahlgren, G. M., Johansson, S. G., \& Brage, T. 1999, AJ, 117, 1454

Moore, C. E. 1971, Atomic Energy Levels, Circular 467, Vol. III (Washington D.C.: US Department of Commerce, NBS, Government Printing Office)

Nagibina. 1958, Opt. Spek., 4, 430

Oertel, G. K., \& Shomo, L. P. 1968, ApJS, 16, 175

Purić, J., Lakićević, I., \& Glavonjić, V. 1980, Phys. Lett. A, 76, 128

Purić, J., Ćuk, M., Dimitrijević, M. S., \& Lesage, A. 1991, ApJ, 382, 353

Sahal-Bréchot, S. 1969a, A\&A, 1, 91

Sahal-Bréchot, S. 1969b, A\&A, 2, 322

Sahal-Bréchot, S. 1974, A\&A, 35, 321

Sahal-Bréchot, S. 1991, A\&A, 245, 322

Sarandev, E. V., Konovalova, C. A., \& Salakhov, M. Kh. 2000, J. Quant. Spectrosc. Radiative Transfer, 67, 110

Tankosić, D., Popović, L.Č., \& Dimitrijević, M. S. 2001, Phys. Scr., 63,54

van Regemorter, H., Hoang Binh Dy, \& Prud'homme, M. 1979, J. Phys. B, 12, 1073

Wiese, W. L., \& Konjević N. 1982, J. Quant. Spectrosc. Radiat. Transfer, 28, 185

Wiese, W. L., \& Konjević N. 1992, J. Quant. Spectrosc. Radiat. Transfer, 47, 185 